\title{
Analysis of Parallel Merge Sort Algorithm
}

\author{
Manwade K. B. \\ Department of Computer Science \& Engineering, Tatyasaheb Kore Institute of Engineering \& \\ Technology, Warananagar, Dist: Kolhapur (MS), India 416113
}

\begin{abstract}
The parallel computing on loosely coupled architecture has been evolved now days because of the availability of fast, inexpensive processors and advancements in communication technologies. The aim of this paper is to evaluate the performance of parallel merge sort algorithm on loosely coupled architecture and compare it with theoretical analysis [1].The parallel computational time complexity is $\mathrm{O}(\mathrm{p})$ [3] using p processes and one element in each process. It has been found that there is no major difference between theoretical performance analysis and the actual result.
\end{abstract}

\section{Keywords}

Parallel computing, Parallel Algorithms, Message Passing Interface, Merge sort, Complexity, Parallel Computing.

\section{INTRODUCTION}

Here, we present a parallel version of the well-known merge sort algorithm. The algorithm assumes that the sequence to be sorted is distributed and so generates a distributed sorted sequence. For simplicity, we assume that $N$ is an integer multiple of $P$, that the $N$ data are distributed evenly among $P$ tasks. Also we have analyzed the performance of the proposed algorithm and it is compared with theoretical analysis.

The sequential merge sort requires $\mathrm{O}(\mathrm{N} \log \mathrm{N})$ [3] time to sort $N$ elements, which is the best that can be achieved (modulo constant factors) unless data are known to have special properties such as a known distribution or degeneracy. This paper describes implementation of the merge sort within a parallel processing environment. In the fully parallel model, you repeatedly split the sub lists down to the point where you have single-element lists. You then merge these in parallel back up the processing tree until you obtain the fully merged list at the top of the tree.

Manuscript received October 5, 2009.

K. B. Manwade was with Department of Computer Science \& Engineering TKIET Warananagar, Kolhapur 416113 INDIA. He is now with the Department of Computer

While of theoretical interest, you probably don't have the massively parallel processor that this would require.

\section{THE PARALLEL ALGORITHM}

This algorithm uses master slave model [4] in the form of tree for parallel sorting. Each process receives the list of elements from its precedor process then divides it into two halves, keeps one half for itself and sends the second half for its successor. To address the corresponding preccedor \& successor we have used the concept of 'myrank multiple'. For a process having odd rank [5] it is calculated as Myrank_multiple=2*Myrank+1;Temp_myrank=Myrank_multip le and for the process having even rank it is calculated as Myrank_multiple=2*Myrank+2;Temp_myrank=Myrank_multip le. It uses recursive calls both to emulate the transmission of the right halves of the arrays and the recursive calls that process the left halves. When the number of processors in the system exhaust then each processor will sort the remaining data. After that it will receive the sorted data from its successor \& merge that two sub lists. Then it sends the result to its precedor. This process will continues up to root node.

procedure parallel_mergesort(DataArray,SizeofData)

Begin

MyData $=$ LeftHalfof[DataArray]

TempData=RightHalfof[DataArray]

Send(TempData)

MyData $=$ Mergesort $($ MyData, $i, j)$

Receive(TempData)

DataArray=MergeResult(MyData,TempData)

End

procedure Mergesort(MyData,i,i)

Begin

If $(j-i>16)$

\{

MergeSort (MyData,i, $(i+j) / 2)$

\} MergeSort $(M y D a t a,(i+j) / 2, j)$

Else

InsertionSort(MyData,i,j)

End

procedure InsertionSort (MyData,i,j)

Begin

//Sequential_InsertionSort

End

\section{THEORETICAL ANALYSIS}

The sequential time complexity is $\mathrm{O}(\mathrm{n} \operatorname{logn})$. In case of parallel algorithm the complexity involves both communication cost and computational cost.

\section{A. Communication}

In the division phase, communication only takes place as follows,

Communication at each step,

$$
\begin{array}{lrl}
\text { Tstartup }+\left(\frac{\mathrm{n}}{2}\right) \text { Tdata } & \mathrm{P} 0 \rightarrow \mathrm{P} 1 \\
\text { Tstartup }+\left(\frac{\mathrm{n}}{4}\right) \text { Tdata } & \mathrm{P} 0 \rightarrow \mathrm{P} 2, & \mathrm{P} 1 \rightarrow \mathrm{P} 3
\end{array}
$$


Tstartup $+\left(\frac{\mathrm{n}}{4}\right)$ Tdata $\mathrm{P} 0->\mathrm{p} 2, \mathrm{p} 1->\mathrm{p} 3$

Tstartup $+\left(\frac{\mathrm{n}}{4}\right)$ Tdata $\mathrm{P} 0->\mathrm{p} 2, \mathrm{p} 1->\mathrm{p} 3$

Tstartup $+\left(\frac{1}{8}\right)$ Tdata $\mathrm{P} 0->\mathrm{p} 4, \mathrm{p} 2->\mathrm{p} 6$,

$\mathrm{p} 1 \rightarrow \mathrm{p} 5, \mathrm{p} 3 \rightarrow \mathrm{p} 1$

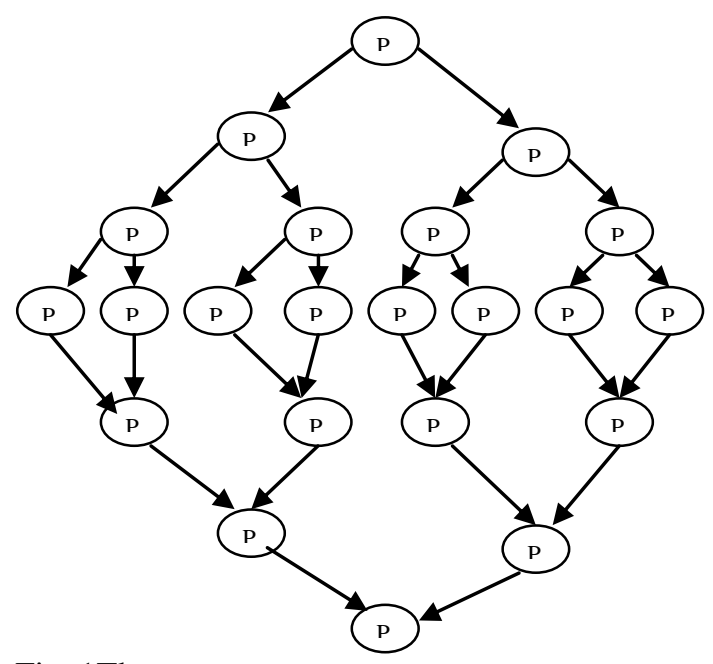

Fig. 1The merge sort tree

With $\log (\mathrm{P})$ steps given $\mathrm{P}$ processors, in the merge phase the reverse communication takes place.

$$
\begin{gathered}
\text { Tstartup }+\left(\frac{\mathrm{n}}{8}\right) \begin{array}{c}
\text { Tdata } \mathrm{P} 4->\mathrm{P} 0, \mathrm{P} 6- \\
>\mathrm{p} 2, \mathrm{P} 5->\mathrm{P} 1, \mathrm{P} 7->\mathrm{P} 3
\end{array} \\
\text { Tstartup }+\left(\frac{\mathrm{n}}{4}\right) \text { Tdata } \mathrm{P} 2->\mathrm{P} 0, \\
\mathrm{P} 3->\mathrm{P} 1
\end{gathered}
$$

Again $\log$ P steps .this leads to the communication time being Tcomm $=2$ (Tstartup $+\left(\frac{\mathrm{n}}{2}\right)$ Tdata

$$
\begin{aligned}
& + \text { Tstartup }+\left(\frac{\mathrm{n}}{4}\right) \text { Tdata } \\
& + \text { Tstartup }+\left(\frac{\mathrm{n}}{4}\right) \text { Tdata } \\
& +\cdots \ldots)
\end{aligned}
$$

\section{$=2(\log \mathrm{P})$ Tstartup $+2 \mathrm{n}$ Tdata}

\section{B. Computation}

Computation only occurs in merging the sub lists merging can be done by stepping through each list, moving the smallest found into the final list first. It takes $2 \mathrm{n}-1$ steps in the worst case to merge two sorted lists each of $\mathrm{n}$ numbers into one sorted list in this manner. Therefore, the computation consists of
Tcomp $=1$
$\mathrm{P0} ; \mathrm{P} 2 ; \mathrm{P} 1 ; \mathrm{P} 3$
Tcomp $=3$
Tcomp $=7$
$\mathrm{P0} ; \mathrm{P} 1$

Hence:

$d=\operatorname{Tcomp}=\sum \log \mathrm{P} i=1(2 \mathrm{i}-1)$

Therefore the total time required is,

Ttotal $=$ Tcomp + Tcomm

$=\sum \log P_{i}=1(2 i-1)+2(\log P)$ tstart

$$
+2 \text { ntdata }
$$

Let us analyze the time by varying the number of processors by keeping $\mathrm{n}=100$,

TABLE I

COMPUTATION TIME REQUIRED FOR MERGE SORTING FOR N=100 BY VARYING NUMBER OF PROCESSORS

\begin{tabular}{|c|c|}
\hline No. of Processors & Ttotal \\
\hline 10 & 203 \\
\hline 20 & 203.60 \\
\hline 30 & 203.95 \\
\hline 40 & 204.20 \\
\hline 50 & 204.39 \\
\hline 60 & 204.56 \\
\hline 70 & 204.69 \\
\hline 80 & 204.80 \\
\hline 90 & 204.91 \\
\hline 100 & 208 \\
\hline
\end{tabular}

Now by keeping the number of processors constant to $\mathrm{P}=10$ \& then varying the number of elements, the time required is,

TABLE II

COMPUTATION TIME REQUIRED FOR MERGE SORTING FOR P=10 BY VARYING NUMBER OF ELEMENTS

\begin{tabular}{|c|c|}
\hline N (No. of elements) & Ttotal \\
\hline 100 & 203 \\
\hline 200 & 403 \\
\hline 300 & 603 \\
\hline 400 & 803 \\
\hline 500 & 1003 \\
\hline 600 & 1203 \\
\hline 700 & 1403 \\
\hline 800 & 1603 \\
\hline 900 & 1803 \\
\hline 1000 & 2003 \\
\hline
\end{tabular}


The graphical representations of the result are as follows,

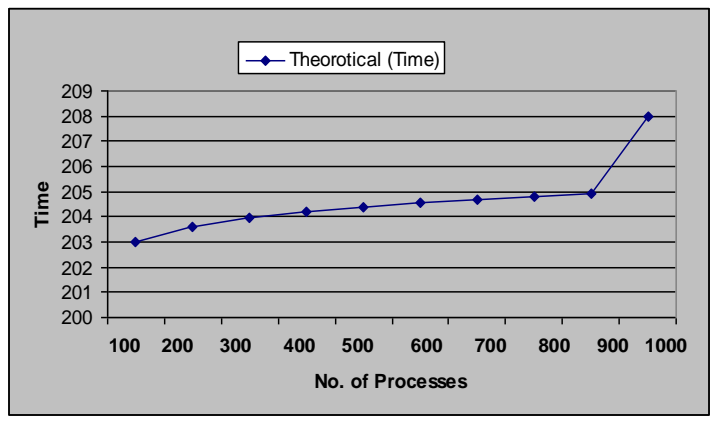

Fig. 2 Theoretical Computation time versus number of processes

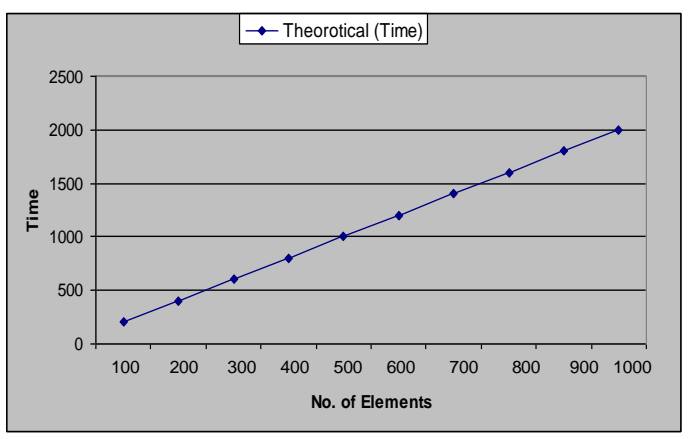

Fig.3 Theoretical Computation time versus number of elements

\section{PRACTICAL ANALYSIS}

We have carried out the experiments in LAN. The computing environment we have used is MPI [4], [5]. To analyze the performance of the algorithm we have used two strategies,

Keep the array size fixed i.e. $\mathrm{N}=100$ and vary the number of processors.

Keep the number of processors fixed i.e. $\mathrm{P}=10$ and vary the size of elements.

We have observed the following results; Table 3 shows the reading for first strategy and Table4 shows the reading for second strategy.

\section{TABLE III}

COMPUTATION TIME (PRACTICAL) REQUIRED FOR MERGE SORTING VARING NUMBER OF PROCESSORS

\begin{tabular}{|c|c|}
\hline No. of Processors & Actual Time (Sec.) \\
\hline 16 & 0.68522 \\
\hline 17 & 0.692969 \\
\hline 18 & 0.73497 \\
\hline 19 & 0.747092 \\
\hline 20 & 0.752514 \\
\hline 21 & 0.825803 \\
\hline 22 & 0.826923 \\
\hline 23 & 0.806718 \\
\hline
\end{tabular}

\begin{tabular}{|l|l|}
\hline 24 & 0.889703 \\
\hline 25 & 0.876956 \\
\hline
\end{tabular}

\section{TABLE IV}

COMPUTATION TIME (PRACTICAL) REQUIRED FOR MERGE SORTING FOR P=10 BY VARYING NUMBER OF ELEMENTS

\begin{tabular}{|c|c|}
\hline N (No. of elements) & $\begin{array}{c}\text { Actual Time } \\
(\text { Sec.) }\end{array}$ \\
\hline 100 & 0.129613 \\
\hline 200 & 0.165852 \\
\hline 300 & 0.17503 \\
\hline 400 & 0.237113 \\
\hline 500 & 0.403223 \\
\hline 600 & 0.438486 \\
\hline 700 & 0.504024 \\
\hline 800 & 0.628536 \\
\hline 900 & 0.65149 \\
\hline 1000 & 0.69522 \\
\hline
\end{tabular}

The graphical representations of the result are as follows,

\section{RESULT}

After plotting the results from table 3 and table4, we have got the following graphs. Figure 4 shows the graph of results for strategy number one, while the Figure5 shows the graph of results for strategy number two.

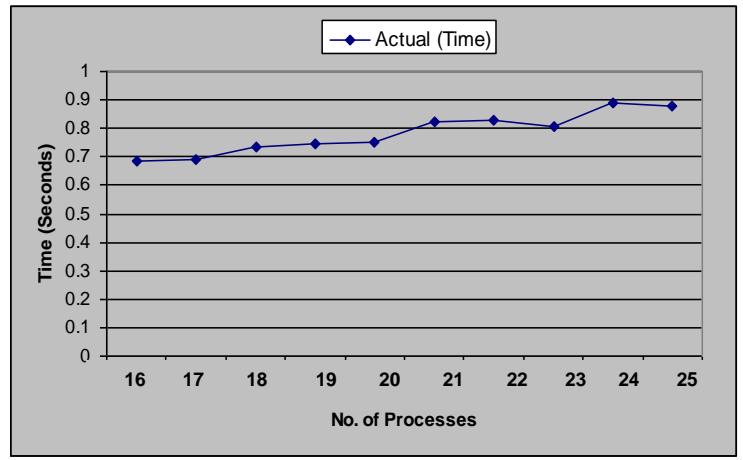

Fig. 4 Actual Computation time versus number of processes 


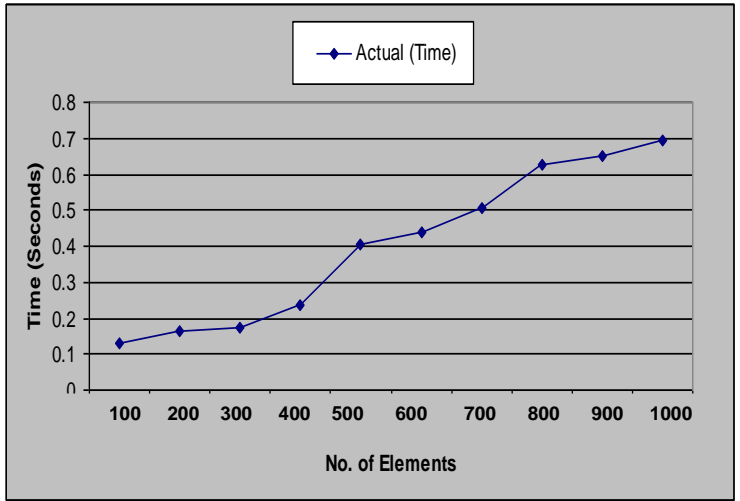

Fig.5 Actual Computation time versus number of elements

\section{CONCLUSION}

The algorithm has been tested on loosely coupled parallel machines and the performance of the algorithm has been observed. It has been found that the computational time of the algorithm varies logarithmically for varying number of processors scenario. Also it is found that for varying number of elements the computational time varies linearly. It is also found that the practical analysis closely matches with theoretical analysis.

\section{REFERENCES}

[1]. K.B.Manwade, R.B.Patil; Parallel merge sort on loosely coupled architecture; National Conference, PSG Coimbator.

[2].Ellis Horowitz, Sartaj Sahani, Sanguthevar Rajasekaran, "Computer Algorithms", Galgotia publication.

[3].Barry Wilkinson \& Michael Charlotte, "Parallel programming techniques and applications using networked workstations and parallel computers", Pearson publication.

[4].http://penguin.ewu.edu/ trolfe/ParallelMerge/ParallelMerge. doc

[5]. Message Passing Interface Forum. MPI: A Message-Passing Interface Standard. Technical Report Version 1.0, University of Tennessee, Knoxville, Tennessee, June 1995.

[6]. The MPI Forum On-line, http://www.mpi-forum.org.

[7].Ananath Grama, Anshul Gupta, "Parallel Computing", Second edition, Addison-Wesley. 